Communications are short papers. Appropriate material for this section includes reports of incidental research results, comments on papers previously published, and short descriptions of theoretical and experimental techniques. Communications are handled much the same as regular papers. Proofs are provided.

\title{
Monochromatic modulation transfer function of the human eye for different pupil diameters: an analytical expression
}

\author{
Pablo Artal and Rafael Navarro \\ Instituto de Optica, Consejo Superior de Investigaciones Científicas, Serrano 121, 28006 Madrid, Spain
}

Received April 28, 1993; revised manuscript received July 20, 1993; accepted July 23, 1993

\begin{abstract}
The modulation transfer function (MTF) of human eyes for different pupil sizes was measured in a group of normal young subjects by use of a double-pass method. Measurements were carried out in the fovea, with monochromatic red light, paralyzed accommodation, artificial pupils, and optimum spherical refraction in each subject. For each pupil size both the averaged MTF and the intersubject variability were calculated. MTF's were approximated by the sum of two exponential functions. This three-parameter function provides a better approximation to our ocular MTF's than do most of the previous parametric models (usually one exponential), especially at intermediate and high spatial frequencies. Further curve fitting of the parameters allowed us to obtain a general expression for the mean MTF as a function of both pupil diameter and normalized spatial frequency. This equation summarizes our estimates of the average monochromatic retinal image quality of the human eye for any pupil diameter with good accuracy and in a compact way, which allows one to incorporate the eye's image quality easily in any particular study.
\end{abstract}

\section{INTRODUCTION}

The retinal image quality of the human eye constitutes a central problem in physiological optics (for a recent review, see Ref. 1), and it has been widely studied also because of its applications to spatial vision, instrumental optics, and optometry, among other areas. The optical performance of the human eye, as the first step in visual processing, is described by the optical transfer function, although often only its modulus, i.e., the modulation transfer function (MTF), is used. Estimates of the MTF of the human eye have been obtained by various methods, both subjective and objective. Among them the double-pass technique has some advantages, in particular its objective nature, its rapidity, and the comfort of the subject during the measurements. Several experiments have been performed recently that validate the double-pass method ${ }^{2,3}$; the results suggest that although double pass tends to underestimate the MTF slightly for high spatial frequencies, it is one of the most suitable and convenient methods in practice. Many ocular MTF results have been obtained, in both polychromatic ${ }^{4}$ and monochromatic ${ }^{5,6}$ light. However, most of those MTF's were obtained only in a few subjects and under different experimental conditions, whereas it is important to have accurate average estimates of the MTF in the human eye as well as of intersubject variability. On the other hand, analytical approximations to the MTF data are useful for easily incorporating the eye's optical degradation in any particular study. In fact, as soon as the first MTF results were measured in the pioneer work of Flamant, ${ }^{7}$ she proposed a simple oneexponential function for approximating the MTF data. Since then, many authors have proposed different expressions to fit experimental data, mainly the polychromatic MTF's of Campbell and Gubisch. ${ }^{4}$ Single-parameter func- tions, such as exponentials ${ }^{7}$ and Gaussians ${ }^{8}$ were proposed first but did not provide a completely accurate fit to the experimental data. More recently, two-parameter approximations (exponential functions) were proposed ${ }^{9}$; they provided a good fit at low and intermediate spatial frequencies but were not so accurate at high spatial frequencies. By incorporation of the pupil diameter as another variable in the analytical expressions, the parametric models are able to describe the MTF for any given pupil diameter. ${ }^{9}$

In this context, our aim in this Communication is to present a simple and accurate parametric model describing the ocular MTF for each pupil size. The MTF was obtained by curve fitting to our new experimental MTF results. We first measured the foveal monochromatic MTF, using a double-pass method ${ }^{6}$ in a group of normal young subjects for different pupil sizes. From the mean MTF results for each pupil, we calculated a compact analytical expression that allowed us to determine the MTF as a function of spatial frequency and pupil diameter.

\section{METHODS}

\section{Double-Pass Technique}

The basic double-pass system is described in detail elsewhere. ${ }^{2,6,10}$ It consists of two stages: (1) the recording of short-exposure coherent aerial images of a point object after double pass through the eye and (2) a digital image processing, including averaging of aerial images, Fourier transform, and computation of the square root to obtain the single-pass ocular MTF.

Control of Optical Factors: Pupil Size, Accommodation, and Centering

The MTF measurements were obtained under controlled conditions of factors potentially affecting the optical per- 


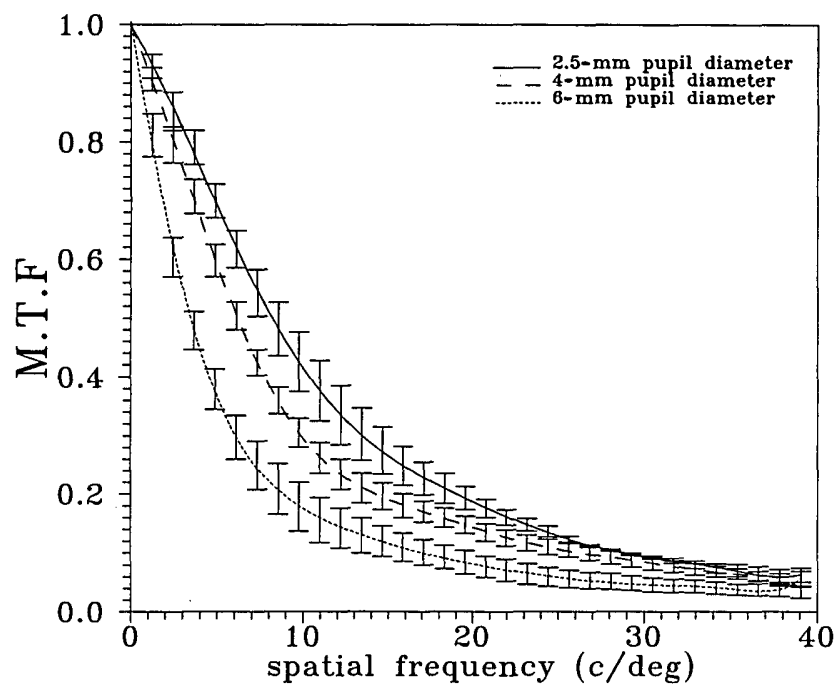

Fig. 1. Average MTF's for a group of normal young subjects for three pupil diameters. Error bars represent intersubject variability in the modulation transfer.

formance: pupil diameter, accommodation, centering, and refractive state. We paralyzed the accommodation by instilling three drops of cyclogyl $1 \%$ in the subjects' eyes with 5-min intervals between drops, starting the measurements 30 min later. Artificial pupils, consisting of an afocal system that imaged a spot (from 1 to $8 \mathrm{~mm}$ in diameter) on the eye's pupil, were used. The use of artificial pupils imposes a careful control of centering during the experiment, since small decentering of the pupil introduces important astigmatism that degrades the retinal image quality. ${ }^{11}$ Objective centering is achieved by use of a control system to monitor the aerial (double-pass) retinal image of the point test in real time by means of an imageintensifier device. This system is based on that proposed by Arnulf et $a .^{12}$ and is described in detail elsewhere. ${ }^{13}$ Assuming that the best (smallest, most symmetrical, and with the highest peak intensity) retinal image corresponds to the centered pupil, we continuously recentered the subject, looking for the optimum position. We also controlled the refractive state by moving a lens until the image monitored by the image intensifier was in optimum focus. Before collecting the data, we determine the best refractive state for each subject with a 4-mm pupil diameter, and this refractive state is maintained constant during the measurements.

\section{Selection of Subjects}

Results were obtained in a group of eight young subjects with mean age of 28 years [2 years standard deviation (SD)]. The subjects were selected after they passed a standard ophthalmological test; we accepted for this study only those subjects with visual acuity $1,(20 / 20)$ or better, with a spherical correction between -2 and $+2 \mathrm{D}$ and less than $0.2 \mathrm{D}$ cylinder refraction. (D stands for diopters.)

\section{RESULTS}

\section{Modulation Transfer Functions for Different Pupil Diameters}

Two-dimensional foveal MTF's were computed from aerial retinal images in all eight subjects for a 4-mm pupil di- ameter and in only four subjects for the other pupil diameters: $1,2.5,3,6$, and $8 \mathrm{~mm}$. Since all the subjects participating in this study are almost astigmatism free, we computed the average radial-profile MTF (averaging over all orientations) for each two-dimensional MTF. Then both the average MTF and the SD of the modulation were computed across subjects for each pupil diameter. Figure 1 presents the average radial-profile MTF's for three pupil diameters $(2.5,4$ and $6 \mathrm{~mm})$ together with the intersubject variability (1 SD) represented by error bars. Figure 2 shows the intersubject variability in the MTF measurements (SD of the modulation) as a function of spatial frequency for the same pupil diameters as shown in Fig. 1. The peak standard deviation is $\sim 0.07$ for $10 \mathrm{c} / \mathrm{deg}$, which is similar for all pupil diameters.

We fitted an analytical expression to the averaged MTF's by a nonlinear least-squares fitting procedure. We chose a three-parameter analytical expression that is the sum of two exponential functions: the function with higher weight and slope fits the low-spatial-frequency range, and the other fits the high-spatial-frequency range; the expression is given by

$$
\operatorname{MTF}(u)=(1-C) \exp (-A u)+C \exp (-B u),
$$

where $A, B$, and $C$ are the fitting parameters ( $A$ and $B$ in degrees; $C$ has no dimensions) and $u$ is the spatial frequency in cycles per degree. The same function has also been used recently by Navarro et al. ${ }^{10}$ to fit off-axis MTF data, while here we apply it to fit the experimental MTF's obtained for different pupil diameters. A sample of the results are shown in Fig. 3, where symbols represent experimental data and curves represent the results of curve fitting to Eq. (1). The resulting set of parameters is summarized in Table 1, along with the rms fitting errors for all pupil diameters. The range of spatial frequency in which Eq. (1) applies is $u$ less than $50 \mathrm{c} / \mathrm{deg}$.

The sum of two exponential functions appears to be an appropriate approximation for the whole family of MTF's. It uses three parameters for each curve and provides a good fit to the complete data set. A direct analytical approximation of the retinal point-spread function (PSF) could be computed from Eq. (1), since the Fourier transform of the sum of two exponential functions is the sum of

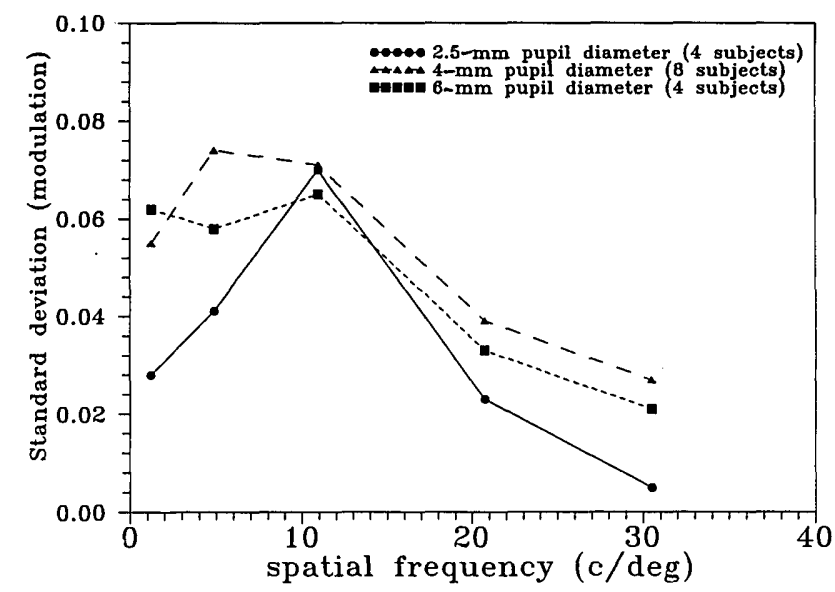

Fig. 2. Standard deviation in the modulation as a function of spatial frequency for the pupil diameters considered in Fig. 1. 


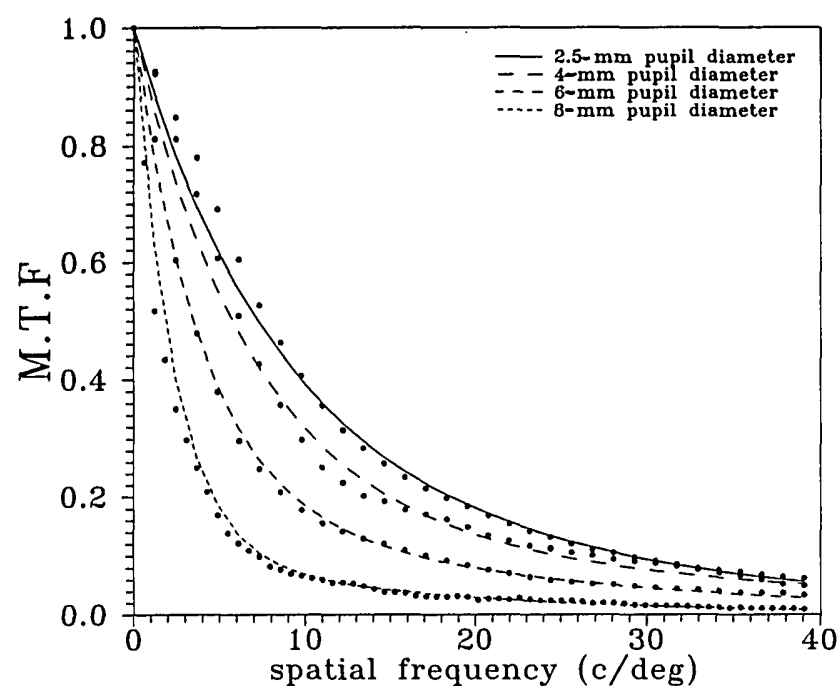

Fig. 3. Average MTF's for four pupil diameters (symbols) and the analytical approximations (curves).

Table 1. Analytical Expression for the Mean MTF for Each Pupil Size ${ }^{a}$

\begin{tabular}{ccccc}
\hline $\begin{array}{c}\text { Pupil Diameter } \\
(\mathrm{mm})\end{array}$ & $\begin{array}{c}A \\
(\mathrm{deg})\end{array}$ & $\begin{array}{c}B \\
(\mathrm{deg})\end{array}$ & \multicolumn{1}{c}{$C$} & rms Error \\
\hline 2.5 & 0.16 & 0.06 & 0.36 & 0.0042 \\
3 & 0.16 & 0.05 & 0.28 & 0.0047 \\
4 & 0.18 & 0.04 & 0.18 & 0.0035 \\
6 & 0.31 & 0.06 & 0.2 & 0.001 \\
8 & 0.53 & 0.08 & 0.11 & 0.0028 \\
\hline
\end{tabular}

${ }^{a} \operatorname{MTF}(u)=(1-C) \exp (-A u)+C \exp (-B u) ; u$, spatial frequency in cycles per degree, $u<50 \mathrm{c} / \mathrm{deg}$. Parameter $C$ has no dimensions.

two Lorentzian functions. However, since the exponentials never reach zero and they do not represent the cutoff frequency, the parametric MTF's yield PSF's that are narrower than the actual PSF's. This implies that one should avoid extrapolating the curve fitting beyond the limits shown in Table 1 or using them to estimate PSF's directly.

\section{Modulation Transfer As a Function of Pupil Diameter and Spatial Frequency}

MTF's are sometimes represented in a spatial-frequency scale normalized to the cutoff frequency for the diffraction-limited system at the same pupil size. This permits comparison of a given optical system to the perfect one. Figure 4 shows MTF's for pupil diameters from 1 to $8 \mathrm{~mm}$ but now plotted against the normalized spatial frequency $\left(u_{0}\right)$. It must be noted that only for the 1-mmdiameter pupil is the eye close to the diffraction limit, while even for the $2.5-\mathrm{mm}$ pupil it appears far from a perfect system. We calculated the new values of parameters in Eq. (1) to fit these MTF's in normalized spatial frequencies. Table 2 presents the values of the new parameters $A_{0}, B_{0}$, and $C_{0}$ and the cutoff spatial frequency for each pupil diameter and wavelength of $632 \mathrm{~nm}$. A further fitting procedure was applied to this new set of parameters, each as a function of pupil diameter. This procedure allows us to represent the whole family of MTF's by a single compact analytical function of two variables: nor- malized spatial frequency $\left(u_{0}\right)$ and pupil diameter $(p)$. Each set of the three parameters listed in Table 2 was fitted to a function of pupil diameter: exponential for parameters $A_{0}$ and $B_{0}$ and linear for parameter $C_{0}$. The resulting final expression of the MTF as a function of normalized spatial frequency $\left(u_{0}\right)$ and pupil diameter in millimeters $(p)$ is given by

$$
\begin{aligned}
\operatorname{MTF}\left(u_{0}, p\right)= & \left(1-C_{1}+C_{2} p\right) \exp \left[-A_{1} \exp \left(A_{2} p\right) u_{0}\right] \\
& +\left(C_{1}-C_{2} p\right) \exp \left[-B_{1} \exp \left(B_{2} p\right) u_{0}\right]
\end{aligned}
$$

where $A_{1}=3.53, A_{2}=0.43, B_{1}=1.69, B_{2}=0.28, C_{1}=$ $0.48, C_{2}=0.037$, and $2<p<8 \mathrm{~mm} ; u_{0}=u / u_{\mathrm{lim}}$, with $u<50 \mathrm{c} / \mathrm{deg}$ and $u_{\mathrm{lim}}$ being the cutoff frequency for a wavelength of $632 \mathrm{~nm}$ (see Table 2).

\section{DISCUSSION}

Retinal MTF's were measured in a group of normal young subjects under controlled conditions of accommodation, pupil centering, and best spherical refractive compensation. The average MTF for each pupil size provides new accurate estimates of retinal image quality. Intersubject variability (see Fig. 2) shows that under controlled conditions the retinal image quality is rather homogeneous among the young subjects that we have measured so far.

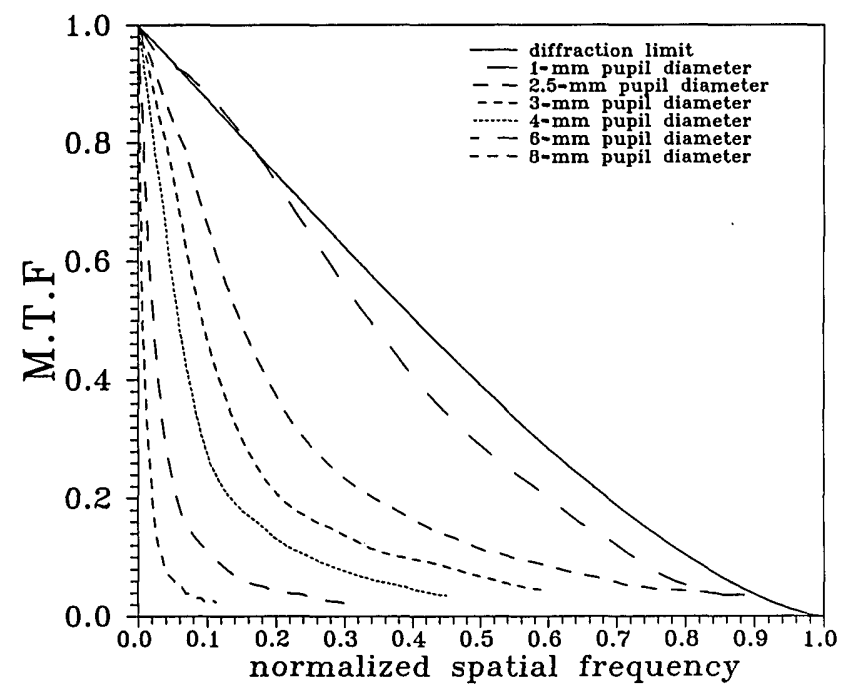

Fig. 4. Average MTF's for six pupil diameters represented in a normalized spatial-frequency scale. (The solid curve corresponds to the diffraction-limited MTF.)

Table 2. Analytical Expression for the Mean MTF for Each Pupil Size ${ }^{a}$

\begin{tabular}{ccccr}
\hline $\begin{array}{c}\text { Pupil Diameter } \\
(\mathrm{mm})\end{array}$ & \multicolumn{1}{c}{$A_{0}$} & \multicolumn{1}{c}{$B_{0}$} & \multicolumn{1}{c}{$C_{0}$} & $\begin{array}{c}u_{\text {lim }} \\
\text { (c/deg) }\end{array}$ \\
\hline 2.5 & 10.57 & 3.96 & 0.36 & 66.1 \\
3 & 12.68 & 3.96 & 0.28 & 79.3 \\
4 & 19.04 & 4.23 & 0.18 & 105.8 \\
6 & 49.19 & 9.52 & 0.2 & 158.7 \\
8 & 112.15 & 16.92 & 0.11 & 211.6 \\
\hline
\end{tabular}

${ }^{a} \operatorname{MTF}\left(u_{0}\right)=\left(1-C_{0}\right) \exp \left(-A_{0} u_{0}\right)+C_{0} \exp \left(-B_{0} u_{0}\right)\left(u_{0}\right.$, normalized spatial frequency; $\left.u_{0}=u / u_{\lim }\right) ; u_{\text {lim }}$, cutoff frequency for the diffractionlimited system calculated for a $632-\mathrm{nm}$ wavelength, with $u<50 \mathrm{c} / \mathrm{deg}$. Parameters $A_{0}-C_{0}$ have no dimensions. 


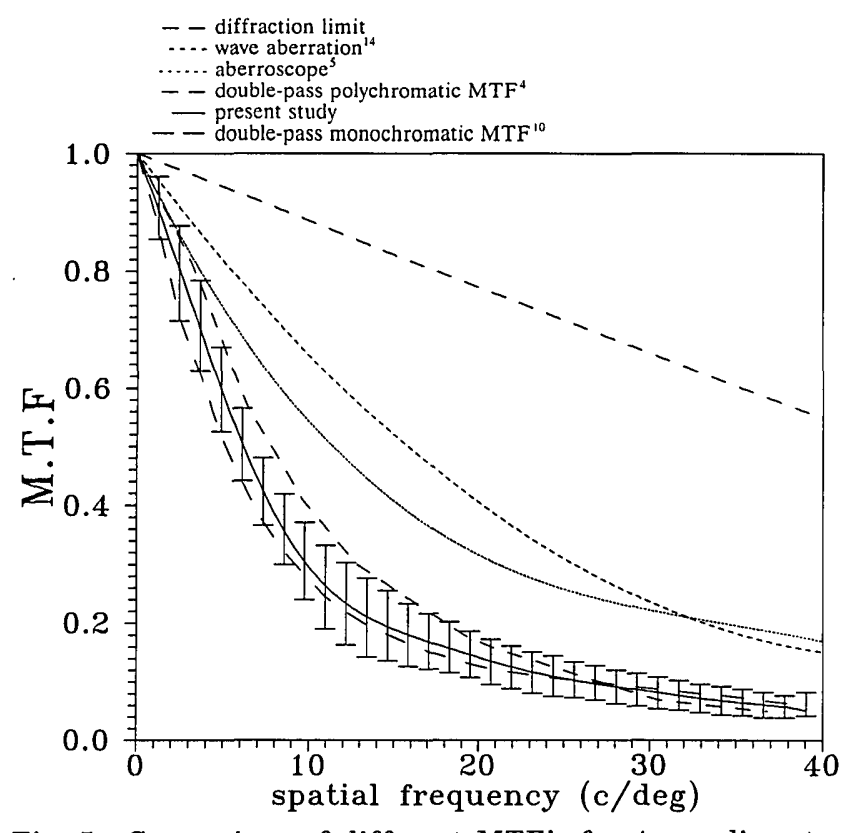

Fig. 5. Comparison of different MTF's for 4-mm-diameter pupil: diffraction limit; best section of the MTF computed from the wave aberration for one subject ${ }^{14} ; 5$-mm-diameter pupil data obtained by an aberroscope method ${ }^{5}$; double-pass polychromatic $\mathrm{MTF}^{4}$; average MTF and error bars (2 SD) obtained in the present study; double-pass monochromatic MTF obtained with natural pupil and accommodation. ${ }^{10}$

Previous studies that used an earlier version of the same apparatus ${ }^{6}$ presented a larger variability in the results, which was due in part to residual astigmatism and nonoptimum spherical refraction in the subjects. A recent study on the effect of aging in retinal image quality ${ }^{13}$ showed a decline in the MTF of older subjects compared with that of younger ones and also a smaller intersubject variability in the MTF's for older subjects. Our present results suggest that the monochromatic image quality in the fovea is rather constant in a young population and that cylinder (astigmatism) and spherical (defocusing) refractions, together with aging, are the main factors causing variability. In particular, aging is a limitation for use of Eq. (2), because MTF's are lower for aged subjects, ${ }^{13}$ and consequently Eq. (2) is not appropriate for subjects older than 60 years.

In the interest of comparing our MTF results with others previously presented in the literature, in Fig. 5 we show a sample of different MTF's for a 4-mm pupil diameter (MTF's for similar pupil diameters have also been included in Fig. 5 when 4-mm pupil data were not available). Our MTF's in eight subjects are represented by the solid curve (average) and error bars (2 SD). Other results obtained by the double-pass technique are similar to our MTF's, although they were obtained in polychromatic light $^{4}$ or with natural accommodation. ${ }^{10}$ However, the production of MTF's either by an aberroscope method ${ }^{5}$ or by computation from the wave aberration (best onedimensional section) ${ }^{14}$ appears to overestimate the MTF considerably with respect to our double-pass results.

The analytical function of Eq. (1), where parameters $A$, $B$, and $C$ depend on pupil diameter, provides the modula- tion in the monochromatic retinal image (for an average normal young eye) for a given spatial frequency. Simpler expressions with only one or two parameters did not fit our data correctly, especially in the higher-spatialfrequency range. This is why we have chosen a somewhat more complicated expression, the sum of two exponential functions, to fit both low-middle- and high-spatialfrequency ranges. Moreover, Navarro et al. used Eqs. (1) and (2) to approximate their experimental MTF's as a function of retinal eccentricity and obtained a good fit. For this reason we believe that the same kind of expression (with different parameters and variables) can be useful in predicting retinal image quality under the most important conditions, such as pupil size, retinal eccentricity, and age.

\section{ACKNOWLEDGMENTS}

The authors thank M. Ferro and I. Miranda for their help with the ophthalmological examination of the subjects. This research was supported by the Comision Interministerial de Ciencia y Tecnología, Spain, under grant TIC91-0438.

\section{REFERENCES}

1. W. N. Charman, "Optics of the human eye," in Visual Optics and Instrumentation, Vol. 1 of Vision and Visual Dysfunction, J. R. Cronly-Dillon, ed. (Macmillan, London, 1991), pp. 1-26.

2. P. Artal and R. Navarro, "Simultaneous measurement of two-point-spread functions at different locations across the human fovea," Appl. Opt. 31, 3646-3656 (1992).

3. P. Artal, R. Navarro, D. H. Brainard, S. J. Galvin, and D. R. Williams, "Off-axis optical quality of the eye and retinal sampling," Invest. Ophthalmol. Vis. Sci. (Suppl.) 33, 3241 (1992).

4. F. W. Campbell and R. W. Gubisch, "Optical image quality of the human eye," J. Physiol. (London) 186, 558-578 (1966).

5. G. Walsh, W. N. Charman, and H. C. Howland, "Objective technique for the determination of monochromatic aberration of the eye," J. Opt. Soc. Am. A 1, 321-328 (1984).

6. J. Santamaría, P. Artal, and J. Bescós, "Determination of the point-spread function of human eyes using a hybrid opticaldigital method," J. Opt. Soc. Am. A 4, 1109-1114 (1987).

7. M. F. Flamant, "Etude de la repartition de la lumière dans l'image retinienne d'une fente," Rev. Opt. 34, 433-459 (1955).

8. H. Kreuger and E. A. Moser, "On the approximation of the modulation transfer function (MTF) by analytical functions," Vision Res. 13, 493-494 (1973).

9. R. J. Deeley, N. Drasdo, and W. N. Charman, "A simple parametric model of the human ocular modulation transfer function," Ophthalmol. Physiol. Opt. 11, 91-93 (1991).

10. R. Navarro, P. Artal, and D. R. Williams, "Modulation transfer of the human eye as a function of retinal eccentricity," J. Opt. Soc. Am. A 10, 201-212 (1993).

11. G. Walsh and W. N. Charman, "The effect of pupil centration and diameter on ocular performance," Vision Res. 28, 659665 (1988).

12. A. Arnulf, J. Santamaría, and J. Bescós, "A cinematographic method for the dynamic study of image formation by the human eye. Microfluctuations of accommodation," J. Opt. 12, 123-128 (1981).

13. P. Artal, M. Ferro, I. Miranda, and R. Navarro, "Effects of aging in retinal image quality," J. Opt. Soc. Am. A 10, 16571663 (1993).

14. P. Artal, "Calculations of two-dimensional foveal retinal images in real eyes," J. Opt. Soc. Am. A 7, 1374-1381 (1990). 